\title{
Integrated production optimisation and monitoring of multi-zone intelligent wells
}

\author{
Reza Malakooti $^{1}\left[\right.$ : Ahmad Zhafran Ayop ${ }^{2} \cdot$ Belladonna Maulianda $^{2} \cdot$ Khafiz Muradov $^{3} \cdot$ David Davies $^{3}$
}

Received: 22 February 2019 / Accepted: 25 June 2019 / Published online: 28 June 2019

(c) The Author(s) 2019

\begin{abstract}
Multi-zone intelligent wells (I-wells) completed with interval control valves and downhole sensors divide the well completion into a number of production intervals that can be managed individually. The production rate from these wells is optimised using either reactive or proactive control strategy. Zonal inflow property values are often used to estimate the zonal multi-phase flow rates (MPFRs) to inform such control strategies. Real-time measurements of the zonal downhole pressure and temperature can be used to estimate the zonal MPFRs, which are considered the main input information to production optimisation algorithms. This paper presents an integrated control and monitoring (ICM) algorithm to maximise production from multi-zone I-wells. The algorithm includes two-level optimisation to design optimum number of required flow tests and optimise either reliability of estimated zonal production (monitoring) or oil production (control). An in-house optimiser has been developed to initiate the required flow tests to perform the ICM workflow, while active soft-sensing algorithm is used to design further flow tests required either to maximise the reliability of estimated zonal properties or maximise oil production. The algorithm was validated using a commercial transient wellbore simulator OLGA ${ }^{\mathrm{TM}}$ in which a five-zone intelligent well was modelled. The simulator results were used as inputs into the ICM algorithm to test the applicability of proposed workflow. Two different workflows of ICM and MPFR were compared in this synthetic case study, and both workflows achieve satisfactory estimates of the zonal properties. However, the ICM workflow attempts to achieve the maximum oil production with a reduced number of flow tests and results in higher cumulative oil production compared to the MPFR workflow. This confirmed that there is a potential to monitor and control zonal production simultaneously with less flow tests in comparison with applying a separate control and monitoring approach. The findings of this study showed it is not necessary to have the accurate estimation of zonal properties in order to maximise the oil production from a multi-zone I-well. The proposed ICM algorithm can also be applied in multi-well flow rate allocation of an interest production system network and optimisation of start-up of multi-zone I-wells.
\end{abstract}

Keywords I-well $\cdot$ Monitoring $\cdot$ Production maximisation $\cdot$ Zonal properties

\section{Introduction}

Wells in heterogeneous formations with high permeability variance exhibit complex downhole multi-phase flow patterns requiring complicated, advanced mathematical

Reza Malakooti

reza.malakooti@port.ac.uk

1 School of Energy and Electronic Engineering, University of Portsmouth, Portsmouth, UK

2 Department of Petroleum Engineering, Universiti Teknologi PETRONAS, Seri Iskandar, Malaysia

3 Institute of Petroleum Engineering, Heriot-Watt University, Edinburgh, UK algorithms to monitor downhole flow rates and optimise the production (Dejam 2018, 2019; Dejam et al. 2013, 2018). Permeability variations across the reservoir sandface may result in excess gas or water production where the highest gas or water flow often occurs through high-permeability zones in the reservoir as shown in Fig. 1. The source of water may be from an aquifer or injected water in a water flooding project, while gas comes from either gas cap or injected gas in a secondary or tertiary recovery method (Olayiwola and Dejam 2019; Rostami et al. 2019; Amirian et al. 2018; Saboorian-Jooybari et al. 2016). The inflow of oil and unwanted fluids (gas and water) from different production intervals within the reservoirs is required to be monitored in order to 
Fig. 1 Schematic of gas and water breakthrough in heterogeneous reservoir (Mathiesen et al. 2014)

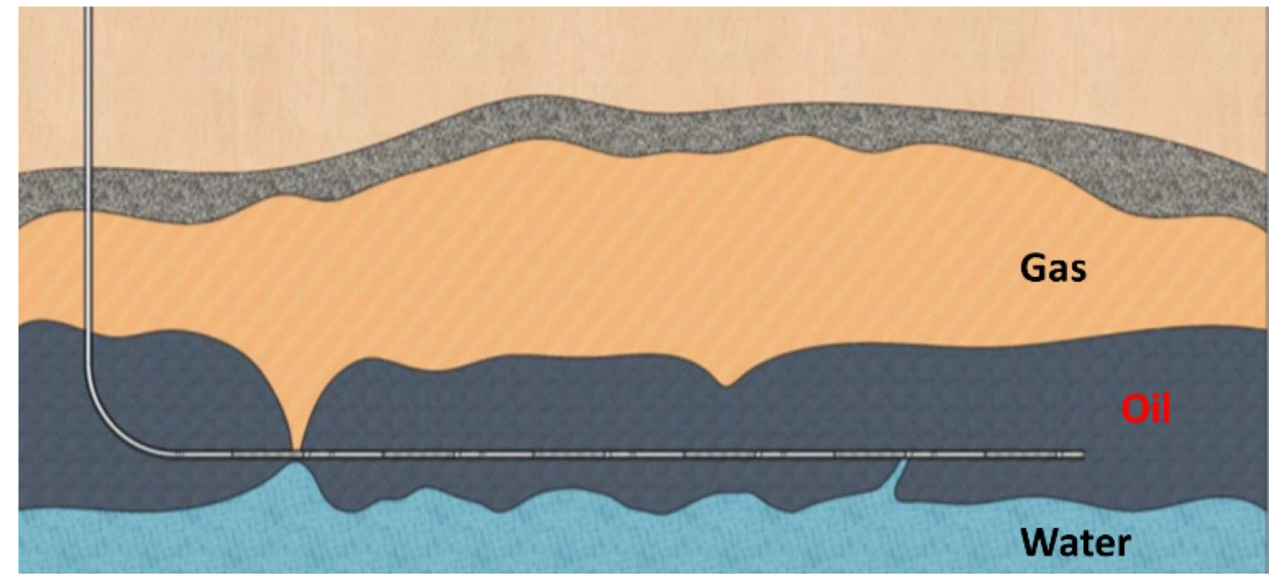

understand and subsequently maximise oil production from these formations.

Intelligent well completion (IWC) provides both downhole sensors and control valves to be installed across individual zones in a heterogeneous formation (see Fig. 2). Downhole sensors measure pressure and temperature across the reservoir sandface and control valves which in turn may be used to estimate zonal inflow rates, while downhole valves such as ICVs control the zonal flow rates and are treated as a control variable in optimisation algorithms to maximise the oil recovery. Figure 2 illustrates a schematic of a multizone I-well including the most interest variables to production engineers to monitor during the well production. The integration of this monitoring task with a control production tool enhances greatly the profitability of the well by minimising the production loss while the well is tested for the monitoring purpose.

The real-time production monitoring and control solutions in multi-zone I-wells have been reported in many publications. The monitoring algorithms are normally based on the concept of soft-sensing technique in which zonal MPFRs are estimated using indirect measurements of temperature and pressure (Nævdal et al. 2005; Leskens et al. 2008; Muradov and Davies 2009; Saputelli et al. 2011). These methods require a multi-phase flow model to relate the measurements to flow rates from individual zones. The soft-sensing estimation of zonal MPFRs may be performed in either passive or active mode. Fixed configuration of the completion is used in passive soft-sensing methods to obtain measurements and estimate the downhole flow rates (Gryzlov et al. 2009; Lorentzen et al. 2010, 2014), while active soft-sensing technique uses optimised sequence of ICV positions in a multi-rate flow tests to estimate zonal MPFRs (Malakooti et al. 2015).

The production control solutions optimise production either with a reactive (Gerebenkin and Davies 2012) or proactive (Brouwer and Jansen 2004; Almeida et al. 2010 Haghighat Sefat et al. 2013) strategy. The production

Fig. 2 Schematic view of a multi-zone I-well

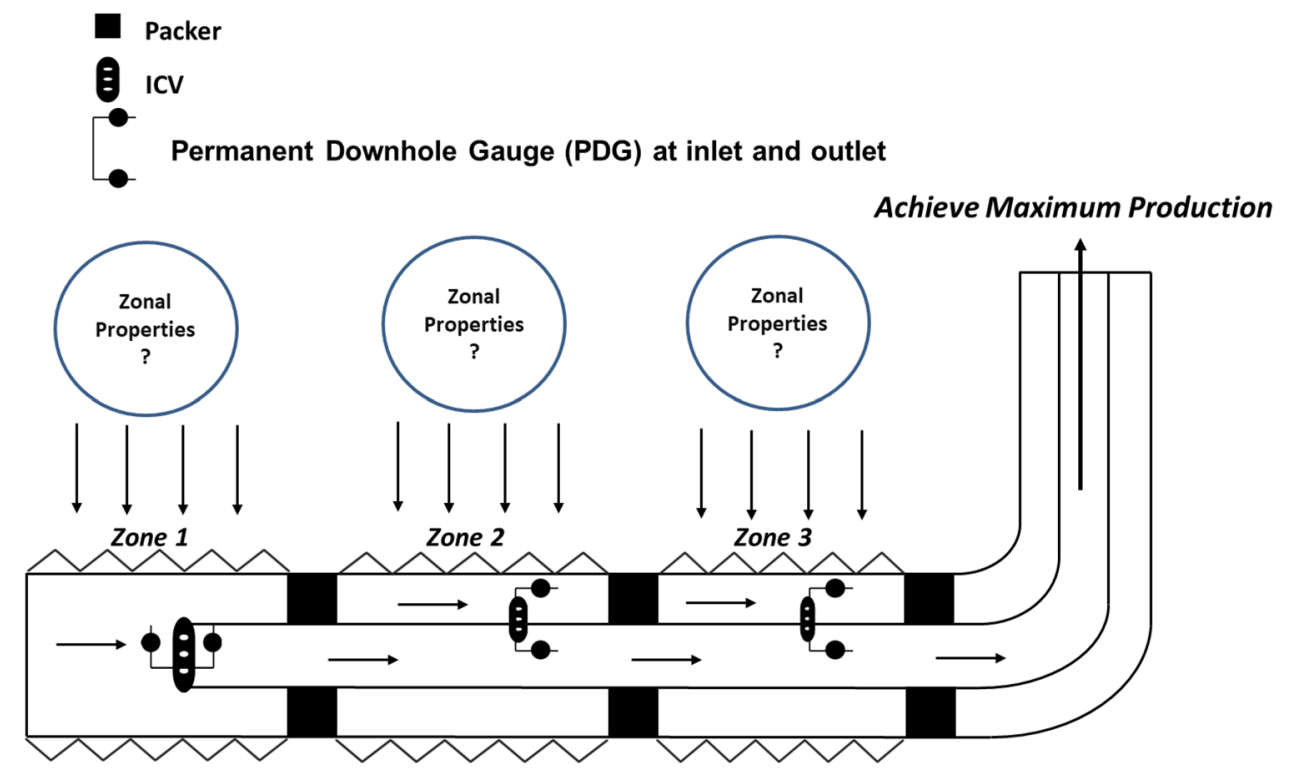


optimisation in a reactive control strategy is instantaneously short term and as such is based on the reservoir and well flow performance at a given time. The proactive production optimisation considers long-term reservoir production.

To the knowledge of the authors of this paper, there is no published study of integrated approach of monitoring and control in hydrocarbon reservoirs except the recent commercialised Manara production and reservoir management system developed by 8 -year collaboration between Saudi Aramco and Schlumberger (Dyer and Bouldin 2016). Manara system presents the industry's first IWC platform to enable simultaneous, real-time monitoring and control of up to 60 compartments in multilateral wells or extended-reach sections longer than $12 \mathrm{~km}$, using a single electric control line (Fig. 3). The system monitors downhole parameters to immediately identify problematic zones, diagnose the cause and adjust downhole valves in real time to optimise the well production. This technology has been successfully applied in two wells in Shaybah oil field of the Rub' al-Khali desert.

Increasingly sophisticated hard and software solutions for monitoring and control have been introduced during the last 30 years to automate and optimise all operational aspects of the production of oil and gas. This paper represents another step in this direction. It describes an integrated control and monitoring (ICM) workflow for optimum design of the sequence of a multi-rate, well flow test in an AWC equipped with ICVs. It provides the well operator with specific ICV settings that will minimise the number of well flow tests to estimate the flow rates of each production zone or lateral with a known accuracy using a test strategy that either maximises the:

1. Reliability of the estimated zonal flow rates (i.e. by focussing on the monitoring objective) or
2. Well's total oil production (i.e. by focussing on the control objective).

This work is the extension of application of active softsensing algorithm (Malakooti et al. 2015) to monitor the zonal flow rates in I-wells. The active soft-sensing approach is different from previous passive soft-sensing studies. The active soft-sensing approach is different from previous passive soft-sensing studies as it designs optimum number of flow tests (measurements) to obtain the most accurate estimate of zonal flow rates. Passive soft-sensing uses indirect measurements from the fixed given configuration of the completion (Lorentzen et al. 2010; Muradov and Davies 2011; Ajayi et al. 2012; Lorentzen et al. 2014). The current study also incorporates the element of production control into the active soft-sensing algorithm and develops a new integrated production monitoring and control workflow. All previous methods proposed to maximise instantaneous production or optimise the well start-up operation are based on availability of zonal properties and known current zonal flow rates (Gerebenkin and Davies 2012; Yusuf et al. 2016; Mukati et al. 2019). However, ICM provides a workflow to achieve these objectives when no information in terms of zonal flow rates is available. The unique design of such a workflow enables production optimisation and eliminates profit loss, while monitoring algorithms are performed to determine the contribution of individual zones into total well production.

This paper first presents the methodology used in this work including the formulation of the problem and the proposed solution strategy to solve it. Secondly, the results from modelling a synthetic five-zone I-well in OLGA ${ }^{\mathrm{TM}}$ and applying both ICM and MPFR workflows on software outputs are provided. Then, the discussion section compares the
Fig. 3 Manara real-time simultaneous monitoring and production control platform (Dyer and Bouldin 2016)

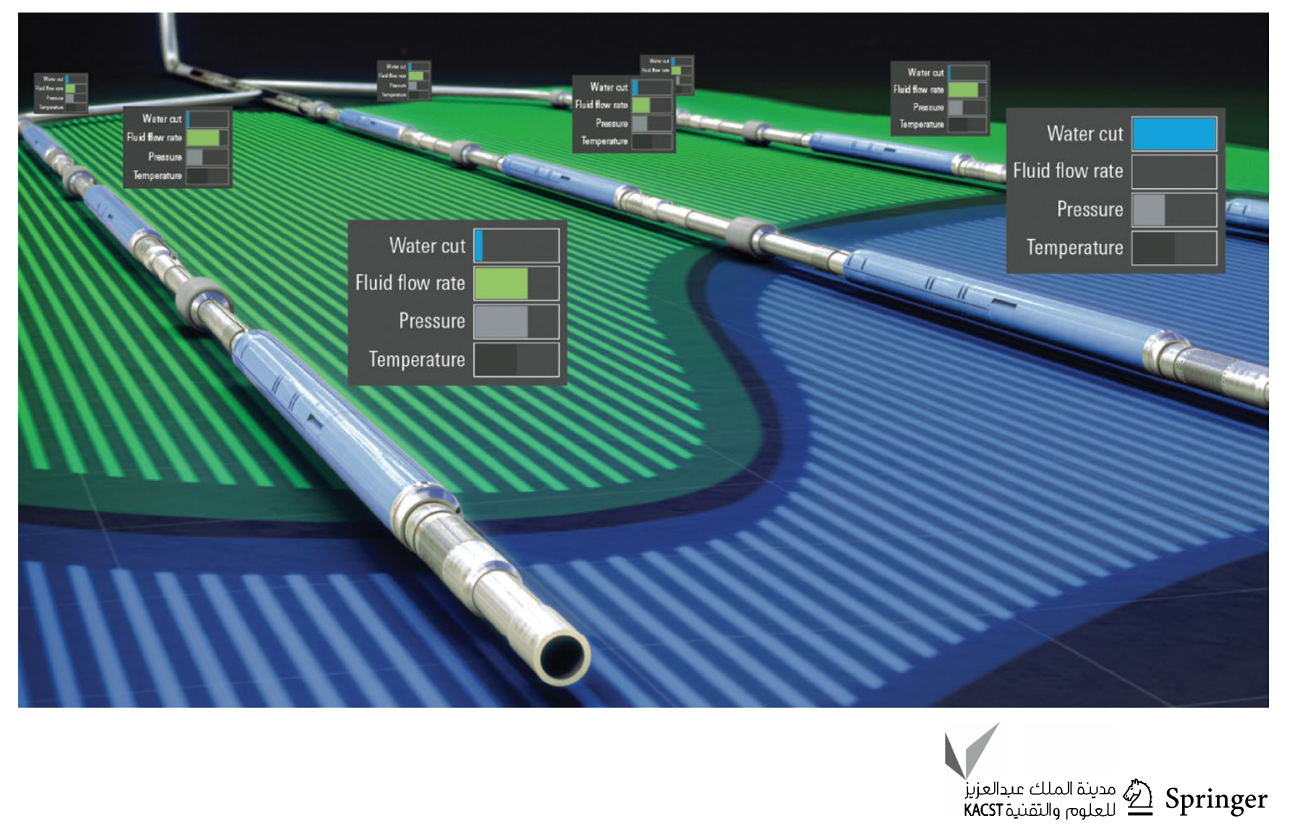


performance of the two workflows to highlight the applications and advantages of ICM approach.

\section{Methodology}

\section{Problem formulation}

This paper presents an ICM algorithm that designs the optimum number of flow tests required to simultaneously monitor the reservoir properties and (reactively) control the well's production. The MPFR, soft-sensing algorithm (Malakooti et al. 2015), was our initial work in this area. The MFPR algorithm analyses use the measured data from an initial $(n+1)$ flow tests in an n-zone I-well to estimate the flow rate and phase fraction of the fluids being produced from each of the of the $n$ zones. These initial flow tests could be either classical, sequential zonal build-up or drawdown flow tests or (not too) historical, routine production data when the zonal flow rate was changed. MFPR algorithm uses deformed configuration simplex optimisation technique to design required next flow tests to estimate most reliable zonal properties. This method is different from simplex linear programming available in Excel Solver.

The ICM concept extends the (Malakooti et al. 2015) MPFR workflow which ensures that the loss in production during the initial $(n+1)$ flow tests is kept to a minimum by a two-stage optimisation workflow:

- Step 1 estimates the zonal properties when the total mismatch of the objective function is minimised.

- Step 2 simultaneously designs the future flow tests while minimising the individual mismatch function (equivalent to the reliability of the estimated zonal properties) or maximising the total oil production.
Both of the ICM strategies use step 1, while step 2 depends on the chosen ICM workflow. We coded the ICM workflow into a Microsoft Excel worksheet with Excel Solver optimising each step's objective function using GRG nonlinear optimiser. Preparation of the worksheet was straightforward, and it provided fast and efficient calculations.

The need for a methodology for designing the initial flow tests becomes increasingly important as the number of zones increases (Skilbrei et al. 2003; Knabe et al. 2014). Zonal pressure build-up or flow rate tests result in production lost; reducing the incentive to carry them out despite being necessary to provide the essential information to efficiently manage the well and/or reservoir production. Pressure depletion, high water-cuts and cross-flow between zones all exacerbate the problem of restarting a zone after a shut-in period if "dead" liquid is present in the tubing because the producing zones were unable to maintain continuous production.

A reactive control (Gerebenkin and Davies 2012) methodology is used for the third and later initial flow tests to ensure the maximum possible oil production is achieved during the flow test. The Excel worksheet follows the steps shown in Fig. 4. The initial $(n+1)$ flow test starts with the well on production, and an arbitrary change is made in the production rate. This provides the data for the Solver to optimise the objective functions. Equation 1 presents the objective function of the first optimisation step (monitoring) that is minimised to calculate the zonal properties. The objective function is based on nodal analysis to predict the well's optimum production rates by simultaneous solution of
Fig. 4 Comparison between optimisation problems

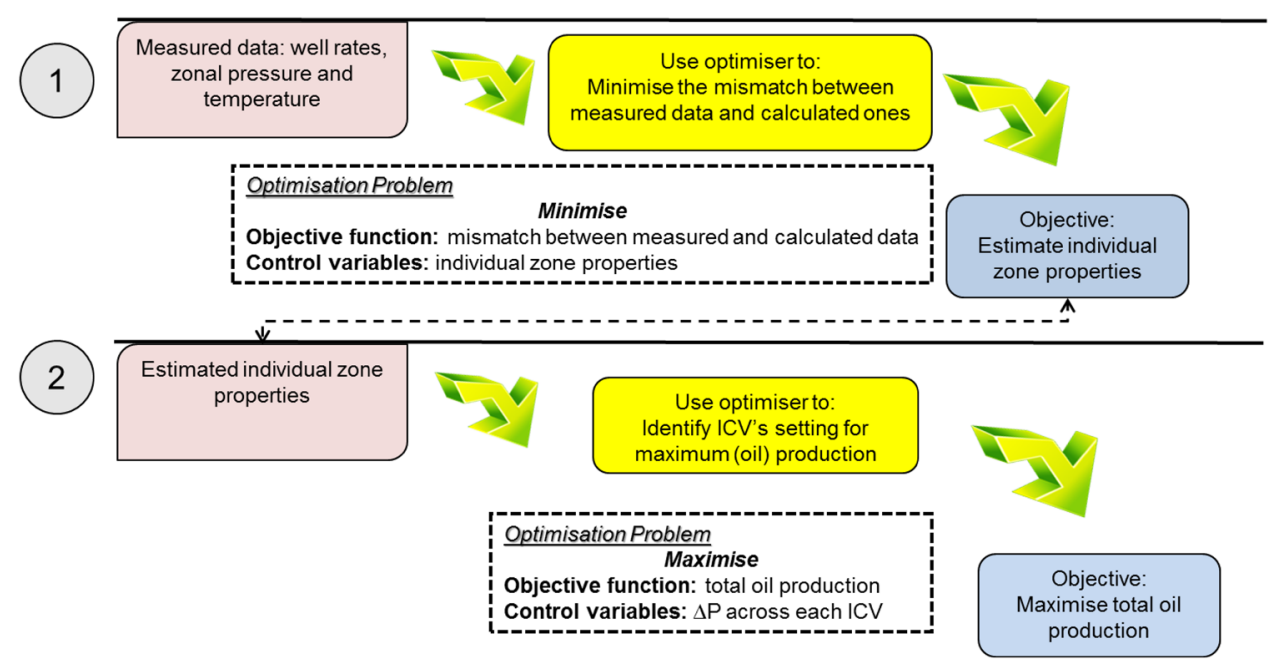


the well's inflow (IPR) and outflow (VLP) equations (Brown and Lea 1985). The heel section of the completion is used as the nodal analysis operating point (see below).

Total Mismatch $=\left(B_{1} \frac{\sum_{i=1}^{K} \operatorname{Mismatch}_{1, i}}{K}\right.$

$$
\begin{aligned}
& +B_{2} \frac{\sum_{i=1}^{K} \operatorname{Mismatch}_{2, i}}{K}+B_{3} \frac{\sum_{i=1}^{K} \text { Mismatch }_{3, i}}{K} \\
& +B_{4} \sum_{j=1}^{n} \frac{\sum_{i=1}^{K} \operatorname{Mismatch}_{4, i, j}}{K}+B_{5} \sum_{j=1}^{n} \frac{\sum_{i=1}^{K} \operatorname{Mismatch}_{5, i, j}}{K} \\
& \left.+B_{6} \frac{\sum_{i=1}^{K} \operatorname{Mismatch}_{6, i}}{K}\right)^{0.5}
\end{aligned}
$$

where $K$ and $n$ are the number of flow tests and zones, respectively. $B_{1}-B_{6}$ represent the weight factors corresponding to each mismatch. (We assumed equal weights of 1 for this study.) The choice of these factors is highly dependent on the measurement's uncertainty. Lower values are assigned to the mismatch parameters involved with erroneous measurements to estimate the most reliable reservoir properties using more accurate measurements.

Equation 1 mismatch parameters are defined by a quadratic function for all the differences between the measured and estimated data as follows:
- Total oil production rate:

Mismatch $_{1}=\frac{\left(Q_{o, \text { measured }}-Q_{o, \text { calculated }}\right)^{2}}{\left(\delta Q_{o}\right)^{2}}$

- Total water production rate:

$\operatorname{Mismatch}_{2}=\frac{\left(Q_{w, \text { measured }}-Q_{w, \text { calculated }}\right)^{2}}{\left(\delta Q_{w}\right)^{2}}$.

- Total gas production rate:

$\operatorname{Mismatch}_{3}=\frac{\left(Q_{g, \text { measured }}-Q_{g, \text { calculated }}\right)^{2}}{\left(\delta Q_{g}\right)^{2}}$

- Pressure drop across ICVs:

$\operatorname{Mismatch}_{4}=\frac{\left(\Delta p_{\text {ICVi,measured }}-\Delta p_{\text {ICVi,calculated }}\right)^{2}}{\left(\delta \Delta p_{I C V i}\right)^{2}}$

- Mixture's average temperature downstream the ICVs:

Mismatch $_{5}=\frac{\left(T_{\text {mix }_{i}, \text { measured }}-T_{\text {mix }_{i}, \text { calculated }}\right)^{2}}{\left(\delta T_{\text {mix }_{i}}\right)^{2}}$

- Zonal build-up pressure drop if any zone is completely closed during a multi-rate flow test:

Fig. 5 A schematic of n-zone I-well

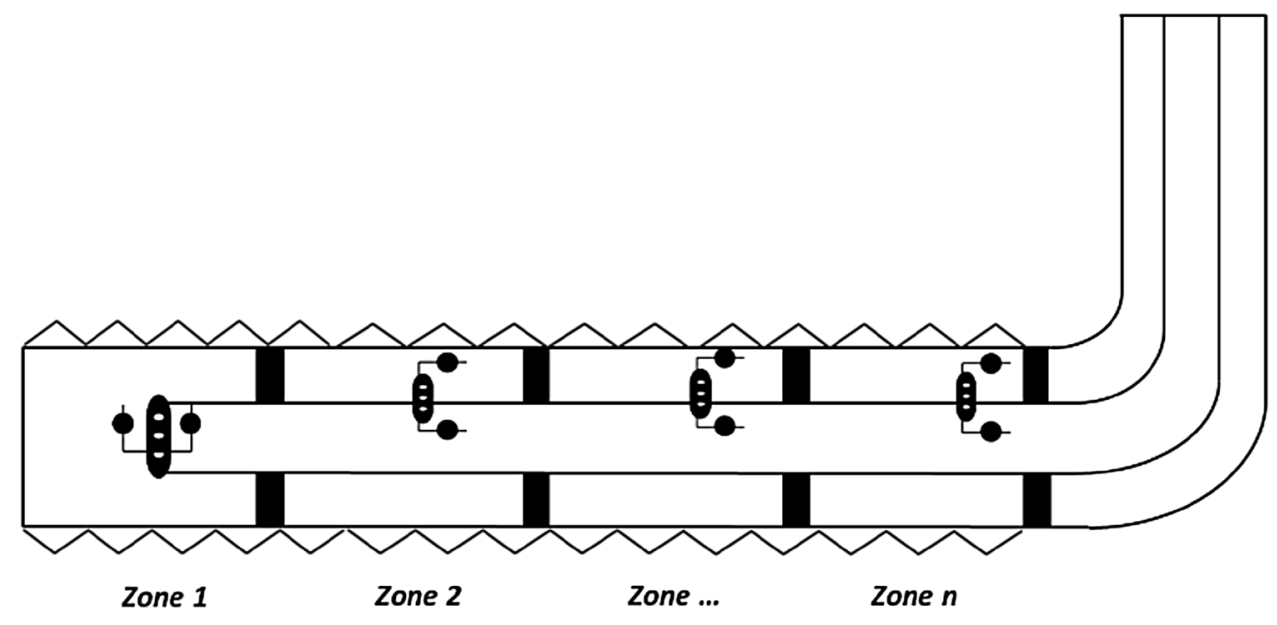

Packer

छ ICV

Permanent Downhole Gauge (PDG) 


$$
\operatorname{Mismatch}_{6}=\frac{\left(\Delta p_{B U i, \text { measured }}-\Delta p_{B U i, \text { calculated }}\right)^{2}}{\left(\delta \Delta p_{B U i}\right)^{2}}
$$

where $i$ represents the measured and calculated values for each zone.

Figure 5 is an n-zone I-well in which the individual zones are characterised by different reservoir pressures (Pr), linear liquid productivity indices (PI), water-cuts (wc) and gas/oil ratios (GOR). These properties are usually unknown to production engineers especially for wells of mature fields and are supposed to be estimated in the monitoring phase of both ICM and MPFR workflows. Regardless of similar or different PVT properties of individual zones, it is assumed this information is available prior to conduct these workflows.

Equations $8-12$ describe the liquid inflow rates versus bottomhole pressure using a nodal analysis approach. The zones at the toe and heel are numbered as 1 and $n$, respectively, and the well inflow rate is calculated using a combination of inflow productivity equations and flow correlations (acceleration terms are ignored).

$Q_{l}=\sum_{i=1}^{n}\left(P I_{l i}\left(P_{r i}-P_{b h p}-\Delta P_{f i n}-\Delta P_{g i n}-\Delta P_{I C V i}\right)\right.$

$Q_{w}=\sum_{i=1}^{n} w c_{i}\left(P I_{l i}\left(P_{r i}-P_{b h p}-\Delta P_{f i n}-\Delta P_{g i n}-\Delta P_{I C V i}\right)\right.$

where $P_{b h p}=$ bottomhole pressure at the heel, $\Delta P_{I C V i}=$ pressure drop across each ICV, $\Delta P_{f i n}=$ pressure drop in the tubing due to friction between zone $\mathrm{i}$ and $\mathrm{n}$ and $\Delta P_{\text {gin }}=$ pressure drop in the tubing due to elevation between zone $i$ and $n$.

Vogel's correlation replaces the linear PI equation when the average reservoir pressure is below the bubble-point.

$Q_{o}=\sum_{i=1}^{n} q_{o_{i} \max }\left(1-0.2\left(\frac{P_{a n_{i}}}{P_{r i}}\right)-0.8\left(\frac{P_{a n_{i}}}{P_{r i}}\right)^{2}\right)$

$Q_{w}=\sum_{i=1}^{n} \frac{w c_{i} q_{{ }_{o_{i}} \max }\left(1-0.2\left(\frac{P_{a n_{i}}}{P_{r i}}\right)-0.8\left(\frac{P_{a n_{i}}}{P_{r i}}\right)^{2}\right)}{\left(1-w c_{i}\right)}$

$P_{a n_{i}}=P_{b h p}+\Delta P_{f i n}+\Delta P_{g i n}+\Delta P_{I C V i}$

The well's outflow expression, or tubing vertical lift performance (VLP), relates the bottomhole pressure to the flow rate through production tubing to the surface. It depends on fluid properties, well depth, tubing size, surface pressure, water-cut and GOR. The most appropriate multi-phase correlation is chosen from many availabe approaches (e.g.
Hagedorn and Brown 1965; Duns and Ros 1963; Beggs and Brill 1973, etc.) to model the VLP.

\section{Problem solution}

Multi-rate flow tests in a multi-zone I-well require changing position of one or more ICVs. Zonal properties are then estimated by a soft-sensing (a.k.a. virtual flow-metering) approach and used to inform the workflow to maximise the oil rate despite the total water-cut ratio from all zones at a given time. Reactive maximisation of oil production requires a methodology that is a combination of nodal analysis and an optimisation algorithm:

Maximise $Q_{o}=f\left(\Delta P_{I C V i}\right)$

Subject to $0 \leq \Delta P_{I C V i} \leq \inf , \quad i=\overline{1, n}$

$0 \leq I C V_{i}$ Opening $\leq 1, \quad i=\overline{1, n}$

The pressure drop across each ICV is treated as the control variable in our algorithm. An interval with a lower and an upper bound is specified to vary the pressure drop between the fully open and the fully closed position. The zero in Eq. 14 indicates a negligible pressure drop across a fully open ICV. The upper bound is no greater than the drawdown when the ICV is fully closed. The calculated optimum pressure drop across the ICV is converted to the ICV opening expressed as a fraction of the maximum flow area. The pressure drop across the ICV, the control variable, is also used to calculate the oil production rate (Eq. 13). The bottomhole pressure and the total oil production rate with these optimum ICV openings are calculated using nodal analysis for each trial flow test. The optimum combination of ICV openings may then be used as the next flow test in the sequence to provide a further set of measurements for updating the zonal property estimates. The choice of objective function is not limited in the ICM workflow as other objective functions such as total water production (total water-cut) or gas production (total GOR) can be formulated to instead solve a minimisation problem.

One benefit of using this paper's optimum design of the multi-rate flow tests for simultaneously estimating the zonal flow rates while maximising the oil production is that all the $(n+1)$ initial flow tests may not always be required. This occurs when if one of the stopping criteria is reached, i.e. when the:

1. Predefined accuracy (or change between flow tests) in the estimated zonal properties is reached or

2. Optimiser proposes no change to the ICV positions while maximising the oil production. 
This approach has been found to be a very efficient procedure for optimising the production while monitoring the zonal properties of an I-well with a large number of zones. However, on some occasions a zonal build-up or drawdown may be preferred to the change in the flow rates discussed above since they can also provide further information on the reservoir properties that are not available when changing the zonal flow rate.

\section{Integrated control and monitoring (ICM) workflow}

Well monitoring is designed to estimate the zonal properties and downhole flow rates, while well control attempts to increase the oil production and reduce the production of unwanted fluids. Accurate estimation of zonal properties is an essential part of optimising the oil recovery from each production interval. The workflow shown in Figure 4 provides a fast and informed production control in multizone I-wells by optimising the ICVs configuration when the zonal properties are unknown. Note that the workflow can be applied to all commingled production systems, including a production network of multiple conventional wells where the wellhead choke setting of individual wells is regulated according to the proposed flow test by ICM workflow to maximise the field production. Simultaneously, the workflow is able to estimate the reservoir properties at each well (i.e. reservoir pressure, productivity index, water-cut, GOR) and allocate the total field oil, water and gas production between the wells.

The right-hand side of Fig. 6 workflow explains the design of required initial flow tests, while the left-hand side presents the active MPFR soft-sensing methodology. The initial flow tests can also use data from a classical drawdown or build-up test on one or more zones, even when the test data are of insufficient quality to provide a meaningful estimate of the zone's reservoir properties with the classical well test equations.

In addition to integrity feature of ICM workflow compared to individual monitoring and control approach, this workflow provides active downhole flow rates monitoring and suggests the optimum well test plan to characterise the well/reservoir properties. The workflow is also based on the optimisation problem in which flexible objective function (e.g. oil rate, unwanted fluid rate, net present value, etc.) can be selected.

\section{Results and discussion}

A synthetic five-zone horizontal I-well model was built in the commercial simulator OLGA ${ }^{\mathrm{TM}}$. This software was selected due to its ability of capturing transient thermal and hydraulic interactions between wellbore and reservoir inflow. The I-well was completed with five ICVs equipped with PDGs to monitor the zonal pressure and temperature. The five zones had significantly different values of

Table 1 Zonal properties of the five-zone I-well

\begin{tabular}{llllll}
\hline Property & Zone-1 & Zone-2 & Zone-3 & Zone-4 & Zone-5 \\
\hline $\begin{array}{c}\text { Reservoir pressure } \\
\text { (psia) }\end{array}$ & 3200 & 3335 & 3625 & 2500 & 3200 \\
$\begin{array}{c}\text { Liquid productivity } \\
\text { index (STB/D/psia) }\end{array}$ & 14 & 12 & 16 & 8 & 8 \\
\begin{tabular}{c} 
Water-cut (fraction) \\
\hline
\end{tabular} & 0.6 & 0.8 & 0.3 & 0.55 & 0.4 \\
\hline
\end{tabular}

Fig. 6 Integrated control and monitoring workflow in multizone I-well

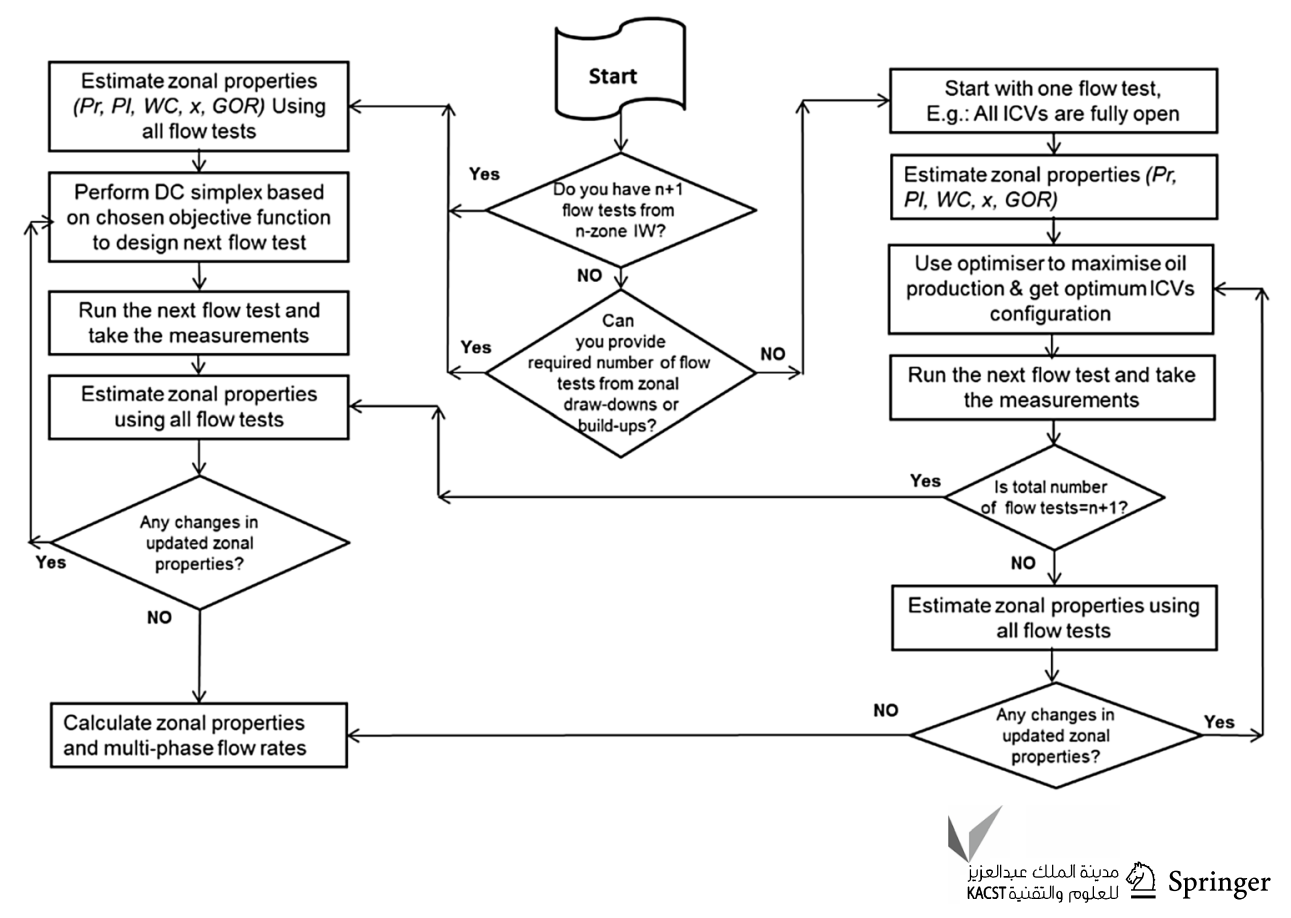




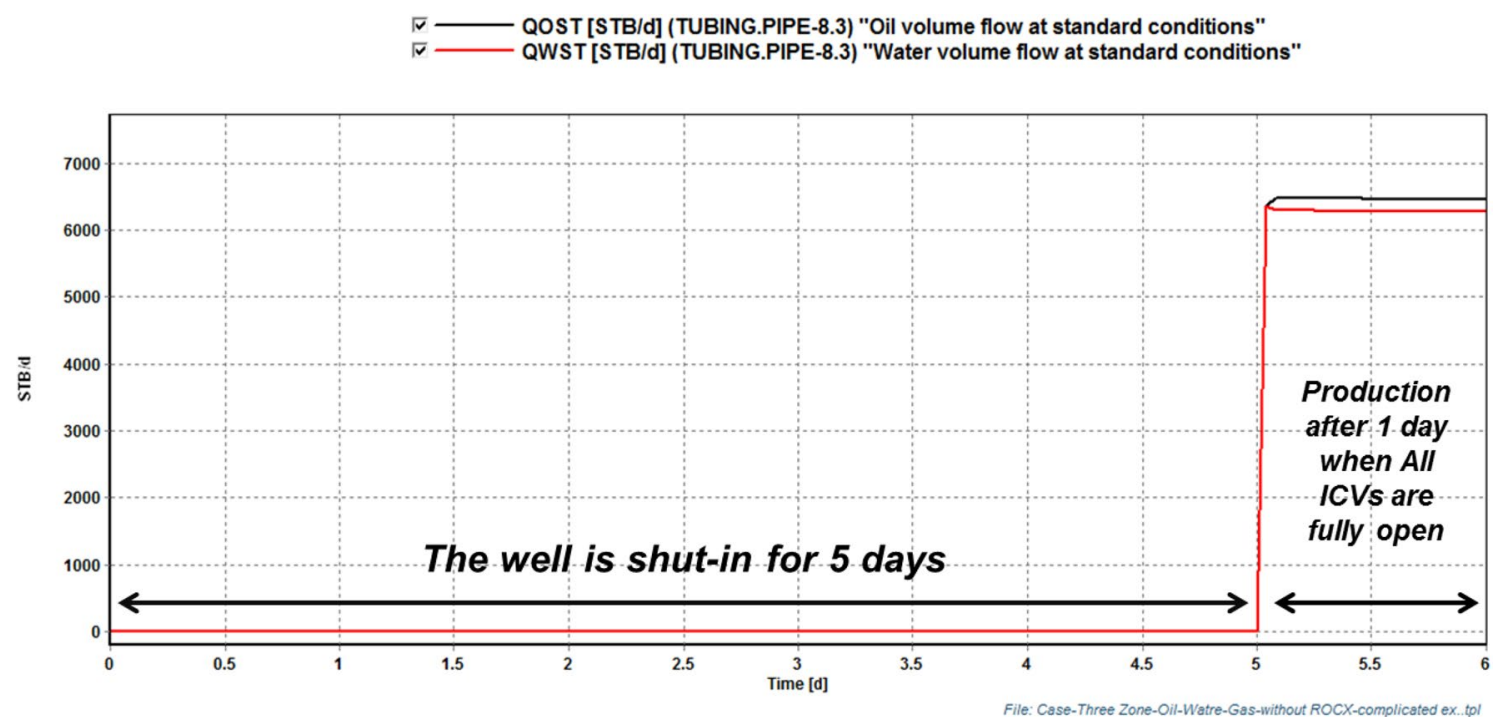

Fig. 7 Total oil and water production from the five-zone I-well

reservoir pressure, liquid productivity indices and water-cuts (Table 1). Zone 2 suffered from a high water-cut, while zone 4's reservoir pressure was sufficiently low that cross-flow occurs in the wellbore when the well is being produced. It was assumed all zones have the same fluid properties. Although only water breakthrough occurs in this synthetic case study, the formulations of fluid flow across the ICVs and wellbore can be modified to include the effect of gas flow rate caused by gas breakthrough. This adds the calculation of in situ gas mass fraction into the algorithm of estimation of zonal properties in addition to zonal reservoir pressure, zonal productivity index and zonal water-cut (Malakooti et al. 2015). Malakooti et al. (2015) also showed the applicability of MPFR workflow in gas reservoirs in which estimation of reliable values of reservoir parameters was difficult due to nonlinearity and extra unknowns in the inflow back-pressure equation. This issue will also happen during the deployment of the ICM algorithm. The measurement of stabilised well bottomhole pressure and flow rates in gas reservoirs may require longer flow tests duration and exhibits additional operational challenges.

Figure 7 shows the well's total oil and water production during a five-day shut-in period followed by one day's production with fully open ICVs. The downhole data measurement system was not working during the shut-in period; hence, the only information available in addition to Fig. 7 on the current zonal properties is that the downhole temperature and pressure measurements are gathered during the production period of one day either side of the zonal ICVs.

The following compares the two options discussed for controlling the well's production. These are the:
Table 2 ICV configuration in multi-rate flow tests

\begin{tabular}{llllll}
\hline Flow test no. & ICV1 & ICV2 & ICV3 & ICV4 & ICV5 \\
\hline 1 & 1 & 1 & 1 & 1 & 1 \\
2 & 0 & 1 & 1 & 1 & 1 \\
3 & 1 & 0 & 1 & 1 & 1 \\
4 & 1 & 1 & 0 & 1 & 1 \\
5 & 1 & 1 & 1 & 0 & 1 \\
6 & 1 & 1 & 1 & 1 & 0 \\
7 & 1 & 0.6 & 0.6 & 0.6 & 0.6 \\
8 & 1 & 0.4 & 0.4 & 0.8 & 0.4 \\
\hline
\end{tabular}

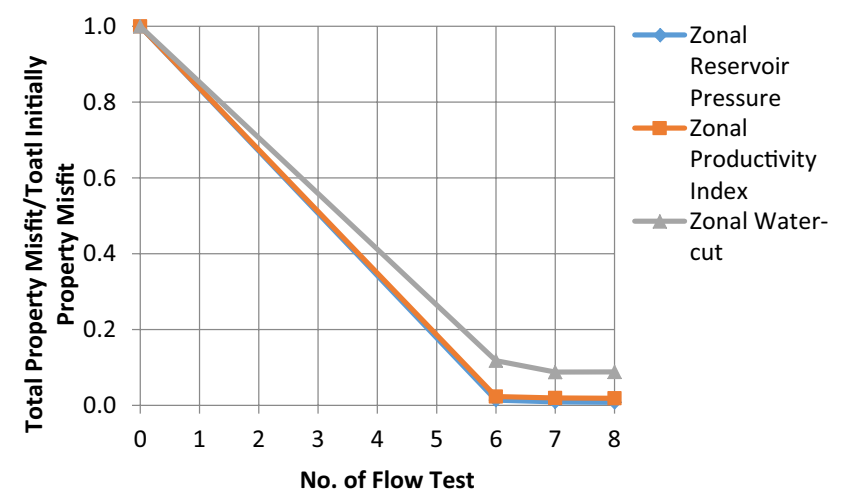

Fig. 8 The total property misfit reduces with an increasing number of flow tests

1. MPFR workflow where active soft-sensing estimates the zonal properties followed by reactive control. This requires designing one extra flow test to maximise the 
oil production (i.e. the monitoring workflow is separated from the control workflow).

2. ICM workflow finds the optimum ICV positions after the 6th day which results in the estimation of unknown zone properties, maximising the total oil production at the same time (i.e. simultaneous monitoring and control workflows).

\section{MPFR workflow}

The deformed configuration (DC) simplex optimisation method (Malakooti et al. 2015) requires $(n+1=6)$ initial flow tests to implement active soft-sensing (Table 2). The seventh and eighth flow tests are used by the DC approach to further increase the accuracy of the estimated zonal properties.

Figure 8 illustrates the convergence trend of zonal properties to their true values. The y-axis is the ratio of total zonal property misfit at each simplex step to the initial total zonal property misfit (Eq. 16):

Total Misfit of Zonal Property

$\overline{\text { Initial Total Misfit of Zonal Property }}$

$$
=\frac{\sqrt{\sum_{i=1}^{n}\left(\frac{\text { estimated value-true value }}{\text { true value }}\right)_{\text {zone }_{i}}^{2}}}{\sqrt{\sum_{i=1}^{n}\left(\frac{\text { Initial value-true value }}{\text { true value }^{2}}\right)_{\text {zone }_{i}}^{2}}}
$$

After the eighth flow test (end of MPFR workflow), reactive optimisation approach is applied to design the next flow test to maximise well oil production. The estimated zonal properties are then used by Excel Solver to design the optimum ICV configuration that maximises the oil production. As expected, the optimiser suggests zones 2 and 4 are closed. Figure 9 illustrates the production

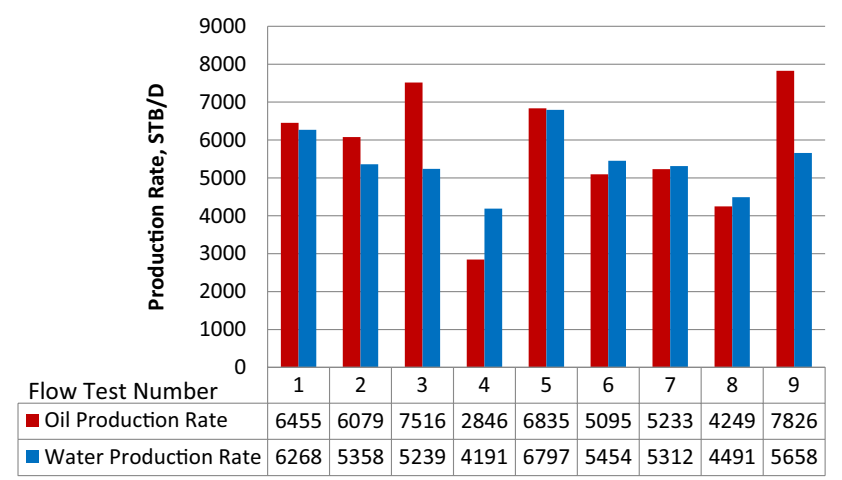

Fig. 9 Total oil and water flow rates for the ninth flow tests required when the control and monitoring steps are separated

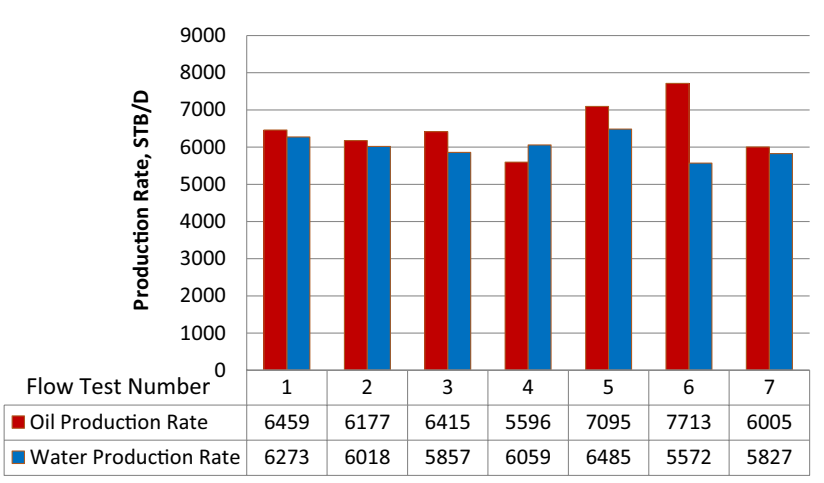

Fig. 10 Total oil and water flow rates for the seventh flow tests required for simultaneous control and monitoring steps

achieved during flow tests $1-8$. The zonal property values have now been estimated as accurately as possible, allowing the maximum oil production to be achieved by the ninth flow test. This is required by the MPFR method since the monitoring workflow is separated from the control workflow.

\section{ICM workflow}

The oil production may reach its maximum value at an earlier flow test if the ICM workflow is applied to the same model (Fig. 10) since the ICM workflow includes maximising oil production as the objective function.

Table 3 lists the designed flow tests and their corresponding ICVs configuration found by the ICM workflow. The ICM workflow was started with two arbitrary flow tests (first and second flow tests). The next four flow tests were designed sequentially by Excel Solver to maximise the oil production, and the seventh flow test is obtained by DC optimisation technique in which the maximum reliability of the zonal properties estimates is considered as an objective function criterion. The objective function criterion of DC algorithm can be selected either from maximum reliability of zonal properties estimates or maximum total oil production. The trend for estimation of the zonal properties is shown in Fig. 11. The ICM workflow stopped by seventh flow test

Table 3 ICVs configuration in multi-rate flow tests

\begin{tabular}{llllll}
\hline Flow test no. & ICV1 & ICV2 & ICV3 & ICV4 & ICV5 \\
\hline 1 & 1 & 1 & 1 & 1 & 1 \\
2 & 0.9 & 0.9 & 0.9 & 0.9 & 0.9 \\
3 & 0 & 1 & 1 & 0 & 1 \\
4 & 1 & 1 & 1 & 0 & 0 \\
5 & 1 & 0.6 & 1 & 0 & 1 \\
6 & 1 & 0 & 1 & 0 & 1 \\
7 & 0.6 & 1 & 1 & 0.8 & 0.6 \\
\hline
\end{tabular}




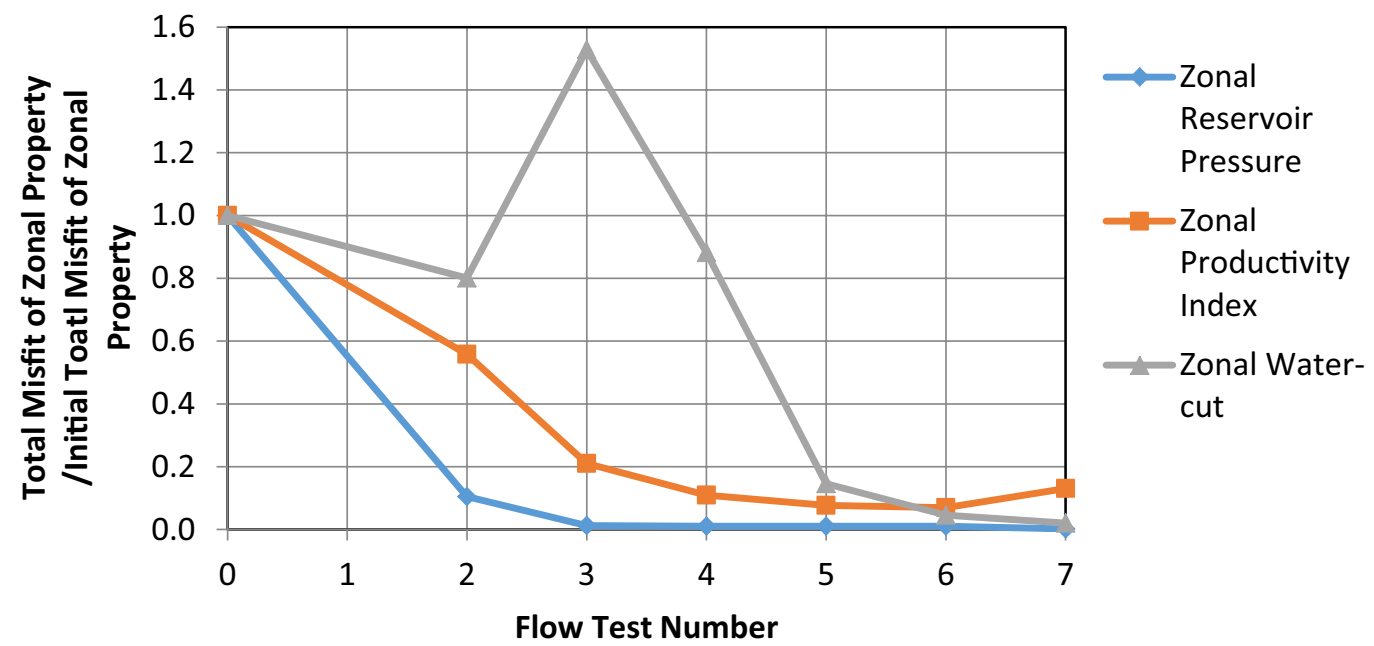

Fig. 11 Estimation of different zonal properties versus flow tests

where no improvement was observed in the estimated zonal properties based on the type of objective function. Similarly, to previous workflow, additional flow test is designed using the reactive control strategy at the end of ICM workflow to maximise oil production rate. This flow test suggests shutting the most problematic zones 2 and 4 which was also obtained earlier in flow test 6 during the implementation of ICM workflow.

\section{MPFR and ICM workflows comparison}

It was observed that both workflows achieve satisfactory estimates of the zonal properties; however, the ICM workflow attempts to achieve the maximum oil production with a reduced number of flow tests and results in higher cumulative oil production compared to the MPFR workflow. This is due to the focus on the production control side in ICM workflow rather than the monitoring of zonal flow rates

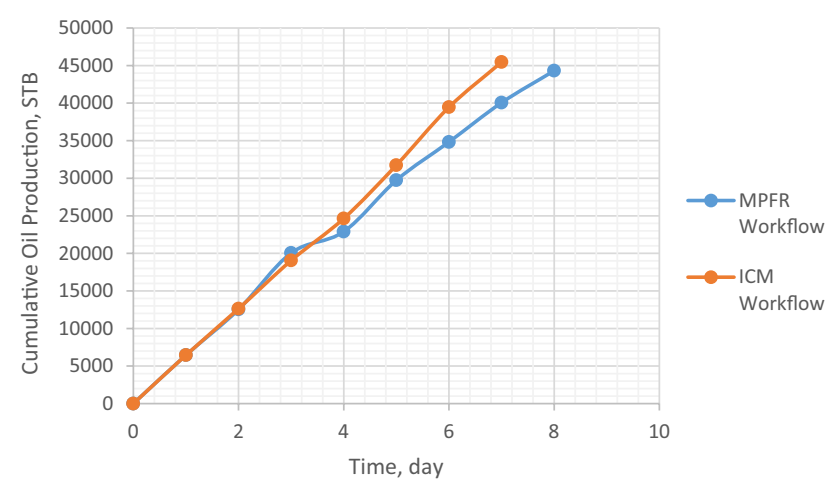

Fig. 12 Cumulative oil production at the end of ICM and MPFR workflows
(Fig. 12). For the synthetic multi-zone case in this paper, the cumulative oil production achieved by ICM workflow was 5000 STB larger than the one by MPFR at the end of day seven. This does not only display the higher production but also decrease in associated costs of implementing further required flow test (manipulating ICVs) to maximise the oil production.

Two different approaches were used to design the multirate flow tests in Tables 2 and 3. Optimisation process (step 1 and 2) shown in Fig. 4 was applied to design the optimum ICVs position in Table 2 where the six initial flow tests were selected manually from the choice of zonal build-up tests together with a flow test in which all zones are fully open and the objective function was maximising the reliability of zonal property estimates. Note that the multi-rate flow tests in Table 3 were obtained using the ICM workflow. As part of this workflow, two arbitrary flow tests are initially chosen and then, the Excel code is used to design the next four flow tests to maximise the oil production. Finally, the optimisation process is continued to design further flow tests in order to maximise the reliability of the zonal property estimates. The choice of arbitrary flow test may not affect the accuracy and convergence of the workflow significantly as long as the selected flow test results in changing zonal tubing and annulus pressure compared to the ones in the previous flow test.

Figures 8 and 11 compare the results between separated control and monitoring workflow (MPFR workflow) and simultaneous control and monitoring approach (ICM workflow). It is shown that more accurate zonal properties are estimated through the MPFR workflow; however, it requires higher number of flow tests to be designed (see Fig. 8). On the other hand, ICM workflow provides lower number of flow tests required to maximise the oil production (Fig. 11) as the Excel code designed the initial flow tests based on 
the oil production maximisation. This approach may result in the estimation of zonal properties with lower accuracies as shown in Fig. 11.

The increase in number of zones increases the number of control variables (to be estimated) in the optimisation problem. This study and our previous work (Malakooti et al. 2015) showed that the algorithm is successful in three-zone and five-zone intelligent wells. However, higher number of flow tests is expected for estimation of reliable zonal properties if number of zones is increased.

In summary, the main advantages of this study are simultaneous implementation of well monitoring and control to mitigate the production loss, no need to access to reservoir properties to optimise production and saving time length to achieve both well monitoring and control objectives. However, there are assumptions within the ICM workflow that may limit its application. All reservoir properties are assumed constant during all the designed flow tests as it is not expected that these parameters change within the couple of days of testing the wells. However, this assumption may be violated at extreme conditions of water/gas coning. Although GRG nonlinear Solver optimiser was used in this study, the ICM workflow can be coupled to other nonlinear optimisation algorithm to investigate the accuracy of estimated zonal properties using such techniques.

\section{Conclusions}

The main remarks of this study are summarised as follows:

- The active multi-phase flow rate monitoring was combined with the production reactive control algorithm to develop the ICM workflow. The ICM workflow not only designs the required initial flow tests of the active softsensing workflow, but also provides simultaneous inflow rate monitoring and oil production control.

- The successful results were reported in this work from the application of ICM workflow to monitor the zonal properties and maximise the oil production for a fivezone I-well.

- The ICV positions are the key variables to achieve the purposes of monitoring and controlling in the ICM workflow. They are regulated to maximise the oil production during $(n+1)$ flow rate tests in n-zone I-wells.

- Additional flow tests by further regulation of the ICVs are designed by the DC optimisation method. DC optimisation provides the flexibility of defining different objective functions such as optimising the ICV positions to achieve either maximum reliability of the zonal properties or maximum oil production.
- Although ICM workflow may not meet monitoring purposes (zonal rates estimation) accurately compared to separate monitoring and control approach, it brings the added value into the well by increasing the cumulative oil production during the flow tests period.

- The ICM workflow provides instantaneous production increase to ensure that minimum production loss occurs during the monitoring and test design in which the production is disturbed by manipulating the downhole valves.

Open Access This article is distributed under the terms of the Creative Commons Attribution 4.0 International License (http://creativeco mmons.org/licenses/by/4.0/), which permits unrestricted use, distribution, and reproduction in any medium, provided you give appropriate credit to the original author(s) and the source, provide a link to the Creative Commons license, and indicate if changes were made.

\section{References}

Ajayi A, Fasasi T, Okuns G (2012) Real time flow estimation using virtual flow measurement techniques: a field application in intelligent well completion. In: SPE 162948, Nigeria annual international conference and exhibition, Lagos, Nigeria

Almeida LF, Vellasco MMBR, Pacheco MAC (2010) Optimization system for valve control in intelligent wells under uncertainties. $\mathbf{J}$ Petrol Sci Eng 73(1-2):129-140

Amirian E, Dejam M, Chen Z (2018) Performance forecasting for polymer flooding in heavy oil reservoirs. Fuel 216:83-100

Beggs DH, Brill JP (1973) A study of two-phase flow in inclined pipes. J Petrol Technol 25(05):607-617

Brouwer DR, Jansen JD (2004) Dynamic optimization of waterflooding with smart wells using optimal control theory. SPE J 9(04):391-402

Brown KE, Lea JF (1985) Nodal systems analysis of oil and gas wells. J Petrol Technol 37(10):1751-1763

Dejam M (2018) The role of fracture capillary pressure on the blockto-block interaction process. J Porous Media 21(11):1121-1136

Dejam M (2019) Advective-diffusive-reactive solute transport due to non-Newtonian fluid flows in a fracture surrounded by a tight porous medium. Int J Heat Mass Transf 128:1307-1321

Dejam M, Hassanzadeh H, Chen Z (2013) Semi-analytical solutions for a partially penetrated well with wellbore storage and skin effects in a double-porosity system with a gas cap. Transp Porous Media 100(2):159-192

Dejam M, Hassanzadeh H, Chen Z (2018) Semi-analytical solution for pressure transient analysis of a hydraulically fractured vertical well in a bounded dual-porosity reservoir. J Hydrol 565:289-301

Duns H, Ros NCJ (1963) Vertical flow of gas and liquid mixtures in wells. In: Paper WPC 10132, presented in 6th world petroleum congress, Frankfurt, Germany

Dyer S, Bouldin B (2016) Technology update: new intelligent completion system enables compartment-level control in multilateral wells. J Petrol Technol 68(09):18-21

Gerebenkin IM, Davies D (2012) A novel optimisation algorithm for inflow control valve management. In: Paper SPE 154472-MS, presented in EAGE annual conference and exhibition incorporating SPE Europec, Denmark

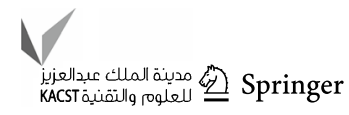


Gryzlov A, Mudde RF, Schiferli W (2009) Inverse modelling of the inflow distribution for the liquid/gas flow in horizontal pipelines. In: Presented in 14th international conference on multiphase production technology, Cannes, France, BHR Group

Hagedorn AR, Brown KE (1965) Experimental study of pressure gradients occurring during continuous two-phase flow in smalldiameter vertical conduits. J Petrol Technol 17(04):475-484

Haghighat Sefat M, Muradov K, Davies D (2013) Field management by proactive optimisation of intelligent wells: a practical approach. In: Paper SPE 167453-MS, presented in SPE middle east intelligent energy conference and exhibition, Dubai, UAE

Knabe SP, Carvajal G, Boisvert I (2014) A smart flow for smart wells: reactive and proactive modes. In: Paper SPE 167821, presented in SPE intelligent energy conference and exhibition, Utrecht, The Netherlands

Leskens M, Kruif B, Belfroid S, Smeulers J, Gryzlov A (2008) Downhole multiphase metering in wells by means of soft-sensing. In: Paper SPE 112046-MS, presented in intelligent energy conference and exhibition, Netherland

Lorentzen RJ, Saevareid O, Nævdal G (2010) Soft multiphase flow metering for accurate production allocation. In: Paper SPE 136026, presented in SPE Russian oil and gas conference and exhibition, Moscow, Russia

Lorentzen RJ, Stordal A, Nævdal G, Karlsen HA, Skaug HJ (2014) Estimation of production rates with transient well-flow modelling and the auxiliary particle filter. SPE J 19(01):172-180

Malakooti R, Muradov K, Davies D, Kuznetsov A (2015) Flow control optimisation to maximise the accuracy of multi-phase flow rate allocation. In: Paper SPE 173873-MS, presented in SPE Bergen one day seminar, Bergen, Norway

Mathiesen V, Werswick B, Aakre H (2014) The next generation inflow control, the next step to increase oil recovery on the Norwegian continental shelf. In: Paper SPE 169233-MS, presented in SPE Bergen one day seminar, Bergen, Norway

Mukati K, Wilson O, Morales M, Arias B, Terry C (2019) Optimization of well start-up using integrated well and electrical submersible pump modelling. In: Paper OTC 29354-MS presented in offshore technology conference, Houston, Texas, USA

Muradov K, Davies D (2009) Zonal rate allocation in intelligent wells. In: Paper SPE 121055-MS, presented in EUROPEC/EAGE conference and exhibition, Amsterdam, Netherlands
Muradov KM, Davies DR (2011) Application of distributed temperature measurements to estimate zonal flow rate and pressure. In: IPTC 15215, international petroleum technology conference, Bangkok, Thailand

Nævdal G, Johnsen LM, Aanonsen SI, Vefring EH (2005) Reservoir monitoring and continuous model updating using ensemble Kalman Filter. SPE J 10(01):66-74

Olayiwola SO, Dejam M (2019) A comprehensive review on interaction of nanoparticles with low salinity water and surfactant for enhanced oil recovery in sandstone and carbonate reservoirs. Fuel 241:1045-1057

Rostami P, Fattahi Mehraban M, Sharifi M, Dejam M, Ayatollahi S (2019) Effect of water salinity on oil/brine interfacial behaviour during low salinity waterflooding: a mechanistic study. Petroleum. https://doi.org/10.1016/j.petlm.2019.03.005

Saboorian-Jooybari H, Dejam M, Chen Z (2016) Heavy oil polymer flooding from laboratory core floods to pilot tests and field applications: half-century studies. J Petrol Sci Eng 142:85-100

Saputelli LA, Oluwole O, Lissanon J, Gonzalez F, Chacon A, Sun K (2011) Inflow performance identification and zonal rate allocation from commingled production tests in intelligent wells-offshore West Africa. In: Paper SPE 146991-MS, presented in annual technical conference and exhibition, Denver, USA

Skilbrei O, Chia R, Schrader K, Purkis D (2003) Case history of A 5 zone multi-drop hydraulic control intelligent offshore completion in Brunei. In: Paper OTC 15191-MS, presented in offshore technology conference, Houston, Texas, USA

Yusuf, R, Thakur, KK, Jama, AA (2016) Integrated dynamic simulation leads to successful start-up of inactive wells through rigless operations. In: Paper SPE 181522-MS, presented in SPE annual technical conference and exhibition, Dubai, UAE

Publisher's Note Springer Nature remains neutral with regard to jurisdictional claims in published maps and institutional affiliations. 